\title{
Longitudinal changes in extent of late gadolinium enhancement in repaired Tetralogy of Fallot: a retrospective analysis of serial CMRs
}

Kwannapas Saengsin ${ }^{1}$, Minmin Lu', Lynn Sleeper ${ }^{1,2}$, Tal Geva $^{1,2}$ and Ashwin Prakash ${ }^{1,2^{*}}$

\begin{abstract}
Background: Right ventricular (RV) late gadolinium enhancement (LGE) occurs due to surgical scarring and RV remodeling, and has been shown to be associated with clinical outcomes in Tetralogy of Fallot (TOF). However, it is not known if cardiovascular magnetic resonance (CMR) LGE extent progresses over time, and therefore, it is not known if serial reassessment of LGE is necessary. We determined the rate of progression in the extent of RV LGE on serial CMR examinations in repaired TOF.
\end{abstract}

Methods: Retrospective review of 127 patients after TOF repair (49\% male, median age at first CMR 18.9 years (Interquartile range (IQR) 13.3,27.0) who had at least two CMRs (median follow-up duration of 4.0 years (IQR 2.1,5.9)) was performed. 84/127 patients had no interventions between serial CMRs (Group 1) while 43/127 patients had transcatheter or surgical intervention between CMRs (Group 2). The extent of RV LGE was assessed using 2 methods: a semiquantitative RV LGE score and a quantitative RV LGE extent expressed as \% of RV mass. Mixed effects linear regression modeling to estimate changes in LGE over time.

Results: RV LGE was present in all patients on the first CMR. \% RV LGE extent and LGE score did not increase over time in either patient group. The mean 5 year rates of change were small and negative for both \% RV LGE extent $[-2.3(95 \% \mathrm{Cl}-2.9,-1.8, p<0.001)$ in Group 1, and $-1.9(95 \% \mathrm{Cl}-3.2,-0.7, p=0.004)$ in Group 2], and RV LGE score $[-0.9(95 \% \mathrm{Cl}-1.1,-0.6, p<0.001)$ in Group 1, and $-0.5(95 \% \mathrm{Cl}-1.1,-0.0, p=0.047)$ in Group 2].

Conclusions: In this serial CMR evaluation of children and adults with repaired TOF, no significant progression in the extent of RV LGE was seen on intermediate term follow-up. Given recent concerns regarding the safety of gadoliniumbased contrast agents, frequent assessment of LGE may not be necessary in follow-up.

Keywords: Tetralogy of Fallot, Late gadolinium enhancement, Cardiovascular magnetic resonance, Longitudinal study

\section{Background}

Despite advances in the surgical, transcatheter, and medical management of Tetralogy of Fallot (TOF), late morbidity and mortality remain unacceptably high, related to chronic pulmonary regurgitation, ventricular

*Correspondence: ashwin.prakash@cardio.chboston.org

${ }^{1}$ Department of Cardiology, Boston Children's Hospital, 300 Longwood Avenue, Boston, MA 02115, USA

Full list of author information is available at the end of the article dysfunction, and ventricular or atrial arrhythmias [1-5]. Right ventricular (RV) late gadolinium enhancement (LGE) on cardiovascular magnetic resonance (CMR) study can occur due to surgical scarring or RV remodeling and has been associated with ventricular dysfunction, arrhythmia, and serum biomarkers of heart failure [6-15]. Although the prognostic significance of LGE in TOF has been previously documented, data regarding LGE progression over time are sparse [16]. Understanding the rate of progression in RV LGE is important original author(s) and the source, provide a link to the Creative Commons licence, and indicate if changes were made. The images or other third party material in this article are included in the article's Creative Commons licence, unless indicated otherwise in a credit line to the material. If material is not included in the article's Creative Commons licence and your intended use is not permitted by statutory regulation or exceeds the permitted use, you will need to obtain permission directly from the copyright holder. To view a copy of this licence, visit http://creativecommons.org/licenses/by/4.0/. The Creative Commons Public Domain Dedication waiver (http://creativeco mmons.org/publicdomain/zero/1.0/) applies to the data made available in this article, unless otherwise stated in a credit line to the data. 
in informing the appropriate frequency of gadolinium administration to assess LGE on follow-up CMRs. This is particularly important in light of recent reports of longterm gadolinium deposits in the brain and other organs $[17,18]$. The purpose of this study was to examine serial CMR data to determine the rate of progression in RV LGE after TOF repair.

\section{Methods}

\section{Subjects}

A retrospective review of all patients with repaired TOF who had undergone 2 or more CMR examinations with LGE imaging between January 2005 and November 2019 was performed. Patients with pulmonary atresia and major aortopulmonary collateral arteries and those with significant imaging artifacts were excluded. Demographic and clinical data were abstracted from electronic medical records. The hospital's Committee on Clinical Investigation waived the requirement for informed consent.

\section{CMR}

All CMR examinations were performed using a wholebody 1.5 T CMR scanner (Achieva, Philips Healthcare, Best, The Netherlands) using standard imaging techniques recommended by the Society for Cardiovascular Magnetic Resonance [19]. Ventricular volumes and ejection fraction, and pulmonary regurgitation fraction were calculated using standard techniques as previously described by our laboratory [20,21]. LGE imaging was performed 10-15 min after intravenous administration of a gadolinium-based contrast agent $(0.15-0.2 \mathrm{mmol} /$ $\mathrm{kg}$ of gadopentate dimeglumine or gadobutrol) using a 2-D phase-sensitive inversion-recovery prepared, fastgradient echo sequence in ventricular long- and shortaxis planes using the following imaging parameters: slice thickness $6-8 \mathrm{~mm}$, slice gap $0-2 \mathrm{~mm}$, reconstructed in-plane spatial resolution between $1.4 \times 1.4 \mathrm{~mm}$ and $1.7 \times 1.7 \mathrm{~mm}$. The short-axis imaging planes were prescribed to cover both ventricles from base to apex. Image analyses were performed using commercially available software (cvi42, v 5.10.1, Circle Cardiovascular Imaging, Calgary, Alberta, Canada).

\section{LGE assessment}

The extent of RV LGE was determined using 2 techniques (Fig. 1): a semiquantitative RV LGE score, and quantitative RV LGE extent expressed as \% of RV mass as follows:

a) Semiquantitative RV LGE score: The score is a modification of a previously described technique [8]. The RV was divided into 9 segments as previously described [7]. Briefly, the RV was first divided into 3 longitudinal regions-basal, midventricular, and apical. Each longitudinal segment was then divided into 3 parts-superior, anterior, and inferior, yielding a total of 9 segments. For each RV segment, LGE extent was graded by a single-blinded observer using a semiquantitative scale as follows: $0=$ No LGE; $1=\mathrm{LGE}$ involving $<1 / 3$ of the segment; $2=\mathrm{LGE}$ involving $1 / 3$ to $2 / 3$ of the segment; $3=$ LGE involving $>2 / 3$ of the segment (Fig. 1). The maximum possible RV LGE score for each subject was 27. Septal insertion point LGE was excluded from RV LGE for this analysis.

b) Quantitative RV LGE Extent (\%): RV endocardial and epicardial contours were drawn manually on the stack of short-axis LGE images, avoiding epicardial fat. Areas of LGE were identified visually and then a semi-automated thresholding technique was applied until the visually identified areas of LGE were marked as areas of positive LGE (Fig. 1). Percentage RV LGE extent was calculated as a sum of the RV mass exhibiting positive LGE, expressed as a percentage of total RV mass. Septal insertion point LGE was excluded from RV LGE for this analysis.

\section{Statistical analysis}

Comparisons among independent groups were performed using Student's t-test, analysis of variance, Wilcoxon rank-sum test or Kruskal-Wallis test, or a Fisher exact test, as appropriate. Spearman correlation was used to estimate the magnitude of association between LGE score and \% LGE extent. To evaluate whether change scores in CMR outcomes differed from zero, a onesample t-test and Wilcoxon signed-rank test were used. Mixed effects linear regression (fixed time, random subject) was used to evaluate the rate of change in RV LGE over time. For this study, assuming a standard deviation of 2.0 for the change in RV LGE score between 2 CMRs and a 2 -sided 0.05 -level test, with 40 subjects in each of 2 independent groups, there is $80 \%$ power to detect a mean group difference of 1.0 between the mean RV LGE change scores. Intra-rater and inter-rater reliability were assessed using the intra-class correlation coefficient (ICC). A p-value of less than 0.05 was considered significant. Analyses were performed using SAS (version 9.4, SAS Institute, Inc., Cary, North Carolina, USA) and $\mathrm{R}$ (version 3.5.1, R Foundation for Statistical Computing, Vienna, Austria).

\section{Results}

Subjects

Patient characteristics are summarized in Table 1. A total of 269 CMR examinations were analyzed on 127 subjects with a median follow-up duration of 4.0 years 


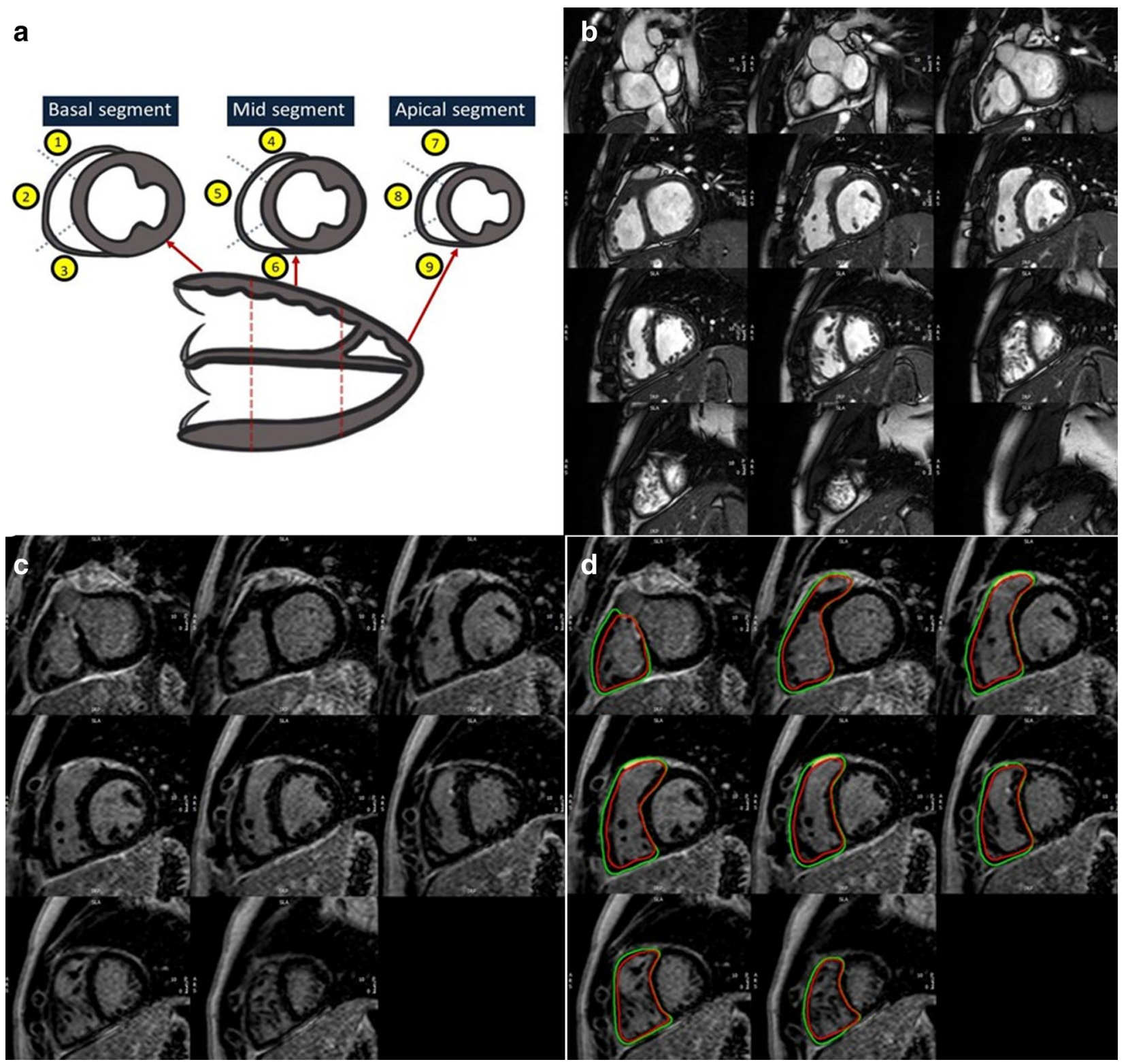

Fig. 1 RV LGE Assessment. a RV LGE score: RV divided into 9 segments. Each segment graded as follows: 0 —no LGE; 1 - LGE involving $<1 / 3$ of segment; 2 - LGE involving 1/3-2/3 of segment; 3-LGE involving $>2 / 3$ of segment (maximum possible score 27) $\mathbf{b}$ Example of cine ventricular short axis in diastolic phase c Example of LGE imaging in the ventricular short axis. $\mathbf{d} \%$ RV LGE Extent: Areas of LGE highlighted using semi- automated manual thresholding. \% RV LGE Extent $=$ (RV LGE mass/Total RV mass) $\times 100$

(IQR 2.1,5.9) between initial and latest CMR examinations. Among the study cohort, 112 subjects $(88 \%)$ had 2 CMR examinations, and 15 (12\%) had 3 CMR examinations available for analysis. The study sample consisted of children and adults, a majority of whom had a transannular patch during their initial surgical repair, which was performed most commonly during the first year of life. 84/127 subjects had no interventions between serial CMRs (Group 1), while 43/127 subjects had transcatheter or surgical intervention between CMRs (Group 2). The types of interventions performed in Group 2 are summarized in Table 1.

\section{RV LGE presence, location, and extent}

RV LGE was present in all patients on the first CMR examination in both groups. The location of LGE was a basal superior segment in 127 subjects (100\%), at the mid superior segment in $82(65 \%)$, and at the mid 
Table 1 Patient Characteristics on Initial CMR

\begin{tabular}{llcc}
\hline & Group 1 (n=84) & Group 2 (n=43) & $\mathbf{p}$-value \\
\hline Male & $41(48.8 \%)$ & $26(60.5 \%)$ & 0.261 \\
Age at repair (years) & $0.7(0.3,5.0)$ & $0.5(0.3,2.9)$ & 0.507 \\
Type of repair & & & 0.184 \\
Valve sparing & $15(18.9 \%)$ & $33(76.7 \%)$ & $3(7.0 \%)$ \\
Transannular patch & $52(61.9 \%)$ & $5(11.6 \%)$ \\
RV-to-PA conduit & $7(8.3 \%)$ & $14.8(12.7,27.8)$ \\
Unknown & $10(11.9 \%)$ & $15.6(13.0,28.7)$ \\
Time since repair (years) & $18.9(13.3,27.0)$ & $19.9(12.6,31.8)$ \\
Age at first CMR, years & $20.4(13.2,34.2)$ & \\
Age at last CMR, years & $25.6(17.8,39.6)$ & $37(86 \%)$ \\
Type of intervention between CMRs & N/A & $6(14 \%)$ \\
Surgical pulmonary valve replacement & N/A & 0.179 \\
Transcatheter pulmonary valve replacement & N & 0.067 \\
\hline
\end{tabular}

Variables are expressed as either frequency (\%) or median (interquartile range). Group 1-patients with no interventions between serial CMRs; Group 2-patients with transcatheter or surgical interventions between CMRs. CMR cardiovascular magnetic resonance; $R V$-to-PA right ventricle to pulmonary artery. $N / A$ not applicable

anterior segment in 16 subjects (13\%) (Fig. 3). There was good correlation between RV LGE score and \% RV LGE extent in both groups $(\mathrm{r}=0.63$ in Group 1, $\mathrm{r}=0.80$ in Group 2; $p<0.001$ for both). Intra-observer reliability in measuring \% RV LGE extent was good (ICC 0.83) in a subset of $40 \mathrm{CMR}$ examinations that were reanalyzed at a later date by the same blinded observer (KS). Inter-observer reliability was modest (ICC 0.6), assessed in a subset of 20 CMR examinations by a blinded second observer (AP).

\section{Serial changes in RV LGE}

Serial changes in RV LGE score and \% RV LGE extent are summarized in Tables 2 and 3 and in Figs. 2 and 3. There was no increase in RV LGE over time, with a small decline in both LGE parameters over time in both patient groups. Mean 5 yeaar changes in both parameters from linear mixed-effects models are summarized in Table 3. As seen in Fig. 2, RV LGE score and \% RV LGE extent increased over follow-up in a small minority of patients, and remained stationary or declined in most patients.

\section{Comparison between groups}

RV LGE score and \% RV LGE extent showed a similar serial trend of gradual decline over serial CMRs in both Groups 1 and 2, with similar 5 yeaar rates of decline (Table 3). As seen in Fig. 1, in Group 1, no patient showed a serial increase in \% RV LGE extent, while a small minority showed a slight increase in the RV LGE score. In Group 2, although the overall trend was similar to that in Group 1, a somewhat larger number of patients showed an increase in \% RV LGE extent and RV LGE score. In
Table 2 Serial Changes in RV LGE and Functional Parameters

\begin{tabular}{lccr}
\hline & Initial CMR & Latest CMR & p-value \\
\hline Group 1 $(\mathrm{n}=84)$ & & & \\
RVEDVI $\left(\mathrm{ml} / \mathrm{m}^{2}\right)$ & $134.5(114.0,150.0)$ & $133.9(117.1,152.1)$ & 0.013 \\
RVESVI $\left(\mathrm{ml} / \mathrm{m}^{2}\right)$ & $65.7(55.1,81.3)$ & $72.0(60.8,84.9)$ & $<0.001$ \\
RVEF $(\%)$ & $49.3(45.8,54.8)$ & $47.6(41.4,51.5)$ & $<0.001$ \\
PR fraction (\%) & $29.0(7.0,40.0)$ & $33.0(9.0,43.0)$ & 0.002 \\
RV LGE score & $4(3,5)$ & $3(2,4)$ & $<0.001$ \\
RV LGE extent $(\%)$ & $5.5(3.4,8.0)$ & $3.1(2.0,4.6)$ & $<0.001$ \\
RV Mass/BSA, g/m² & $16.6(14.0,19.4)$ & $16.6(14.7,19.0)$ & 0.652 \\
RV Mass:Volume, & $0.12(0.10,0.16)$ & $0.12(0.10,0.15)$ & 0.318 \\
g/ml & & & \\
Group 2 (n=43) & & & $<0.001$ \\
RVEDVI (ml/m²) & $175.7(141.6,200.4)$ & $118.7(102.2,144.3)$ & $<.001$ \\
RVESVI (ml/m²) & $85.6(67.8,106.1)$ & $63.8(51.5,82.8)$ & $<0.013$ \\
RVEF (\%) & $50.1(42.7,55.0)$ & $46.0(40.7,50.5)$ & 0.013 \\
PR fraction $(\%)$ & $46.0(30.0,55.0)$ & $5.0(0.0,13.0)$ & $<0.001$ \\
RV LGE score & $4(2,5)$ & $3(2,4)$ & 0.030 \\
RV LGE extent (\%) & $4.4(2.7,7.7)$ & $3.9(2.6,6.3)$ & 0.009 \\
RV Mass/BSA, g/m² & $19.1(14.9,22.5)$ & $14.7(12.5,16.8)$ & $<0.001$ \\
RV Mass:Volume, & $0.11(0.09,0.12)$ & $0.12(0.09,0.14)$ & 0.018 \\
g/ml & & & \\
\hline
\end{tabular}

Variables are expressed as median (interquartile range). Group 1-patients with no interventions between serial CMRs; Group 2-patients with transcatheter or surgical interventions between CMRs. Median time between initial and latest CMR was 4.3 years (IQR 2.9, 6.6) for Group 1 , and 2.7 years (IQR 1.6 to 5.3 ) for Group 2). BSA body surface area, CMR cardiovascular magnetic resonance, $E D V I$ end-diastolic volume index, ESVI end-systolic volume index, EF ejection fraction, $P R$ pulmonary regurgitation, $L G E$ late gadolinium enhancement, $R V$ right ventricle

this group, the only factor associated with an interval increase in both \% RV LGE extent and LGE score $(n=6)$ was older age at repair $(p=0.009$, supplemental Table 1$)$. 
Table 3 Mixed effects model of longitudinal changes

\begin{tabular}{|c|c|c|c|c|}
\hline \multirow[b]{2}{*}{ Variables } & \multicolumn{2}{|l|}{ Group $1(n=84)$} & \multicolumn{2}{|l|}{ Group $2(n=43)$} \\
\hline & Mean 5 year change $(95 \% \mathrm{Cl})$ & $p$-value & Mean 5 year change $(95 \% \mathrm{Cl})$ & $p$-value \\
\hline RV LGE score & $-0.88(-1.12,-0.64)$ & $<0.001$ & $-0.54(-1.07,-0.01)$ & 0.047 \\
\hline$\%$ RV LGE extent & $-2.34(-2.91,-1.76)$ & $<0.001$ & $-1.92(-3.18,-0.67)$ & 0.004 \\
\hline RVEDVI $\left(\mathrm{ml} / \mathrm{m}^{2}\right)$ & $1.63(-2.37,5.63)$ & 0.421 & $-42.2(-60.0,-24.4)$ & $<0.001$ \\
\hline RVESVI $\left(\mathrm{ml} / \mathrm{m}^{2}\right)$ & $4.07(1.15,6.98)$ & 0.007 & $-19.1(-28.9,-9.27)$ & $<0.001$ \\
\hline RVEF (\%) & $-2.81(-3.92,-1.71)$ & $<0.001$ & $-3.11(-5.97,-0.26)$ & 0.033 \\
\hline PR fraction (\%) & $1.36(-0.04,2.76)$ & 0.057 & $-24.1(-33.4,-14.9)$ & $<0.001$ \\
\hline RV Mass/BSA, $\mathrm{g} / \mathrm{m}^{2}$ & $-0.28(-1.03,0.48)$ & 0.470 & $-3.46(-5.53,-1.39)$ & 0.002 \\
\hline RV Mass:Volume, $\mathrm{g} / \mathrm{ml}$ & $-0.004(-0.01,0.03)$ & 0.243 & $0.01(-0.006,0.03)$ & 0.212 \\
\hline
\end{tabular}

$p$-values $<0.05$ denote that the slope of longitudinal change differs significantly from zero. Group 1-patients with no interventions between serial CMRs; Group 2patients with transcatheter or surgical interventions between CMRs. BSA body surface area, CMR cardiovascular magnetic resonance, EDVI end-diastolic volume index, $E S V I$ end-systolic volume index, $E F$ ejection fraction, $P R$ pulmonary regurgitation, $L G E$ late gadolinium enhancement, $R V$ right ventricle

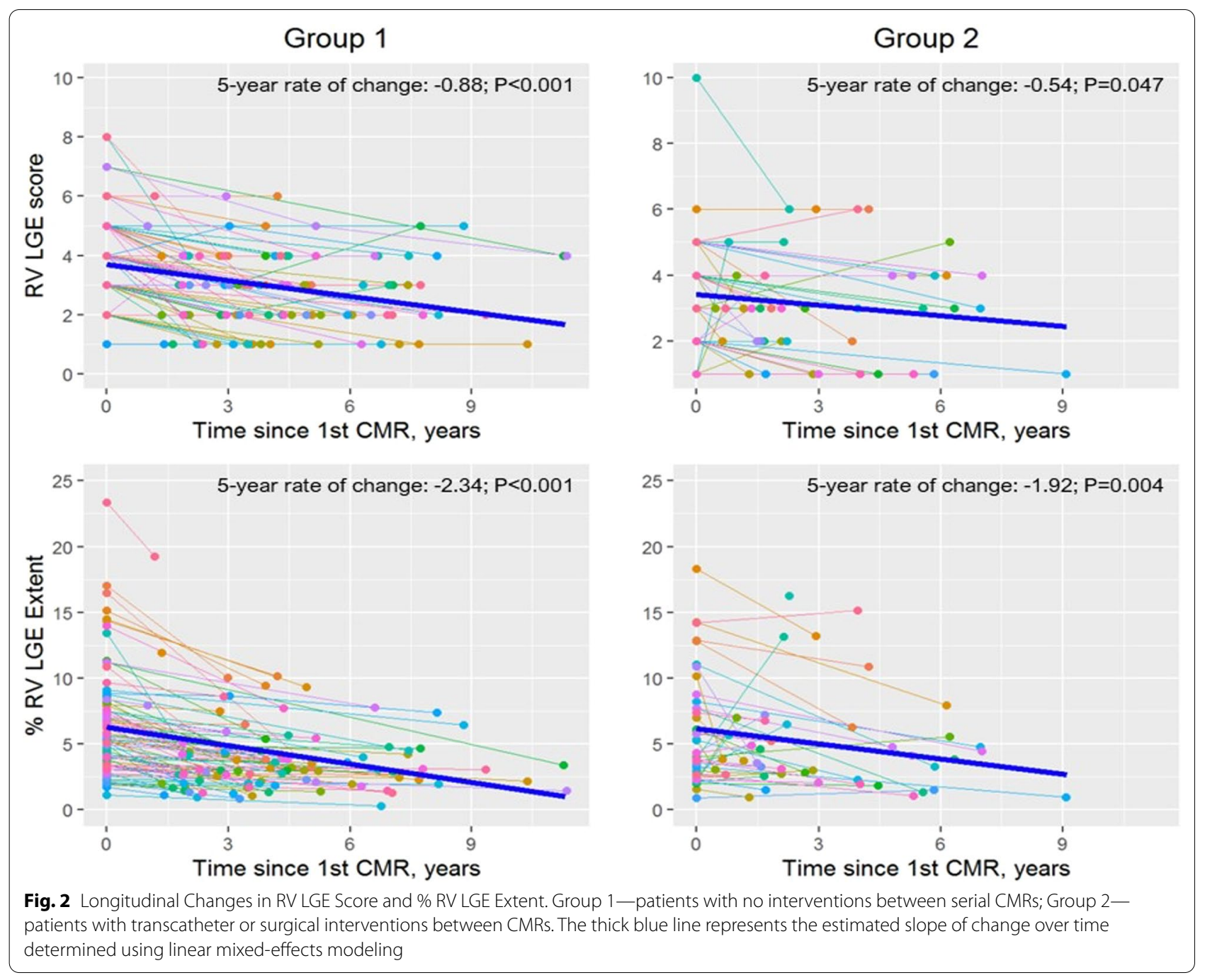




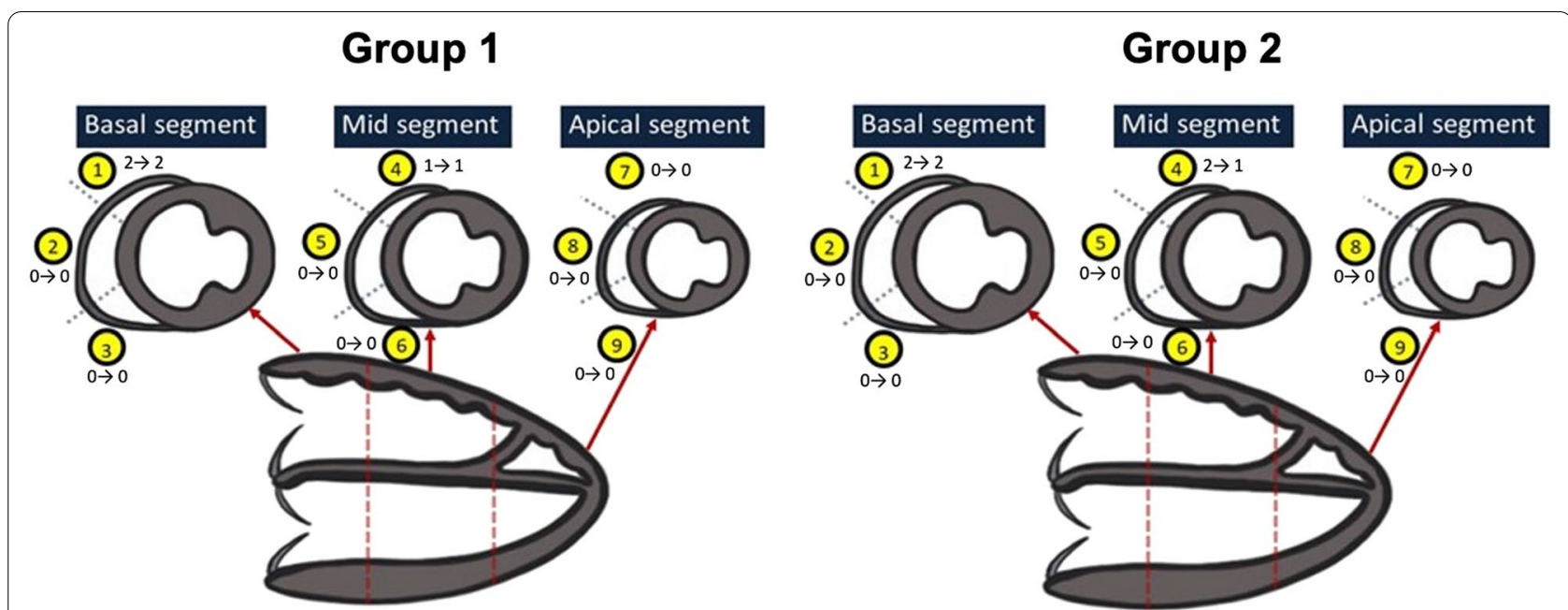

Fig. 3 Longitudinal Changes in Segmental RV LGE Score. Group 1-patients with no interventions between serial CMRs; Group 2-patients with transcatheter or surgical interventions between CMRs. Median baseline and follow-up RV LGE scores for each segment are annotated next to each segment

\section{Factors associated with decline in RV LGE}

We explored the hypothesis that a serial increase in $\mathrm{RV}$ end-diastolic volume indexed to body surface area (RVEDVI) with a corresponding increase in RV mass may lead to a serial decline in RV LGE score or \% RV LGE extent. However, we found no significant association between the rate of change in either parameter of RV LGE, and the rate of change in RVEDVI.

\section{Discussion}

In this serial CMR study of children and adults with repaired TOF, although RV LGE was present in all subjects on the initial CMR, the extent of RV LGE analyzed using 2 measures (LGE score and \% LGE extent) did not significantly increase during follow-up, rather showing a slight decline over serial examinations.

\section{RV LGE-patterns and serial changes}

The patterns of RV LGE in our cohort were similar to those previously reported, with the most common site for LGE being the RV outflow tract. [6] Several prior studies have identified the presence and extent of RV LGE as a risk factor for adverse long-term outcomes in TOF patients $[2,12,13,22]$. However, the serial rate of change in the RV LGE extent has not been previously studied. Ylitalo et al. [23]. found in a cross-sectional study that the extent of RV LGE is higher in patients with a longer duration of follow-up and they hypothesized that LGE extent increased over time. However, the results of our longitudinal study suggest a lack of significant progress in RV LGE extent during a median follow-up period of 4 years. Further, we found no correlation between the extent of
RV LGE and changes in RV volumes during follow-up. It is possible that the finding of a higher LGE extent in patients with a longer duration of follow-up in prior studies may reflect a higher extent of RV surgical scarring, possibly related to an older surgical era. The strengths of the current analysis are its longitudinal design and the quantitative assessment of RV LGE using 2 methods by a single observer.

\section{Comparison between groups}

In the present analysis, we evaluated 2 groups of patients-those free of intervention between serial CMRs and those who had surgical or transcatheter intervention between serial CMRs. We analyzed these groups separately, since surgical manipulation of the outflow tract, as well as artifact caused by implanted valves could potentially confound the serial assessment of LGE extent. The findings of a similar lack of progression in RV LGE in both groups suggests that RV LGE in TOF patient possibly represents fixed post-surgical scarring that is not significantly modulated by long term volume loading due to pulmonary regurgitation, or by intervening procedures, at least in the intermediate term (median follow-up duration was 4 years).

\section{LGE assessment}

There are inherent difficulties in quantifying RV LGE due to the thin free wall. We used a previously described method for calculation a semiquantitative RV LGE score, in addition to a new method to quantify RV LGE extent [8]. In using the second method for quantifying RV LGE extent, we avoided techniques that automatically 
designate areas of LGE using a pre-defined standard deviation cutoff relative to normal myocardium, as this can be fraught with error in the thin-walled RV. Instead, we manually identified areas of RV LGE and then quantified the extent of RV LGE in these areas. We found good correlation in the extent of RV LGE calculated using both methods and longitudinal trends in RV LGE were also similar using both techniques. We found good inter-rater and modest inter-observer reliability in quantifying \% RV LGE extent. Good inter-rater reliability in assessing the RV LGE score has been previously reported [8].

\section{Clinical implications}

Our results have important clinical implications. Since we did not observe a significant increase in RV LGE over a median follow-up duration of 4 years, this suggest that RV LGE does not progress rapidly. Therefore, frequent serial assessment of LGE may not be necessary, thereby avoiding the frequent administration of gadoliniumbased contrast agents in these patients, in light of several reports of long-term retention of gadolinium in patients who receive multiple doses of contrast $[17,18]$. It should be noted that our findings apply to patients in a more recent surgical era.

\section{Limitations}

Several limitations of the current work are worth considering. First, there are known limitations related to its retrospective single-center design. However, these are likely offset by the use of serial longitudinal data using a consistent imaging protocol and analysis by a single observer. Second, while we used serial CMRs, the median duration of follow-up was 4 years and therefore we are not able to exclude progression in RV LGE over longer periods of time. Studies with larger numbers of patients with a longer duration of follow-up may help answer this question more definitively. Third, due to its retrospective design, we did not have high resolution 3-D LGE imaging or imaging for diffuse fibrosis available for serial assessment, as these techniques have only become available more recently. It is possible that follow-up studies using these more sensitive techniques may detect progression in RV fibrosis. Finally, we did not have sufficient numbers of subjects with LV LGE to analyze longitudinal changes in LV LGE over time.

\section{Conclusions}

In children and adults with repaired TOF, the RV LGE extent does not increase significantly based upon serial CMRs during a median follow-up of 4 years. Given recent concerns regarding long-term gadolinium retention, our data do not support the routine frequent serial assessment of LGE in TOF patients.

\begin{abstract}
Abbreviations
CMR: Cardiovascular magnetic resonance; ICC: Intraclass correlation coefficient; IQR: Interquartile range; LGE: Late gadolinium enhancement; PA: Pulmonary artery; PR: Pulmonary regurgitation; RV: Right ventricle/right ventricular; RVEDVI: Right ventricular end-diastolic volume index; RVEF: Right ventricular ejection fraction; RVESVI: Right ventricular end-systolic volume index; TOF: Tetralogy of Fallot.
\end{abstract}

\section{Acknowledgements \\ None}

Authors' contributions

KS designed the study, analyzed the CMR images and drafted and revised the manuscript; ML performed statistical analyses; LS helped with study design, and supervised the statistical analyses; TG provided guidance during study design and data analysis and helped revise the manuscript; AP was overall incharge of the study, designed the study, supervised CMR image analysis, and revised the manuscript. All authors read and approved the final manuscript.

\section{Funding}

Dr. Saengsin's research fellowship was funded by the Chiang Mai University Hospital, Chiang Mai, Thailand.

\section{Availability of data and materials}

The datasets generated and/or analysed during the current study are not publicly available, as this was not approved by the hospital's Committee on Clinical Investigation for this study.

\section{Declarations}

Ethics approval and consent to participate

The Department of Cardiology's Scientific Review Committee approved the study, and the Hospital's Committee on Clinical Investigation waived the requirement for informed consent.

\section{Consent for publication}

Not applicable.

\section{Competing Interests}

The authors declare that they have no competing interests.

\section{Author details}

${ }^{1}$ Department of Cardiology, Boston Children's Hospital, 300 Longwood Avenue, Boston, MA 02115, USA. ${ }^{2}$ Department of Pediatrics, Harvard Medical School, Boston, MA, USA

Received: 25 February 2021 Accepted: 5 May 2021

Published online: 21 June 2021

\section{References}

1. Stirrat J, Rajchl M, Bergin L, Patton DJ, Peters T, White JA. High-resolution 3-dimensional late gadolinium enhancement scar imaging in surgically corrected Tetralogy of Fallot: clinical feasibility of volumetric quantification and visualization. J Cardiovasc Magn Reson. 2014;16:76.

2. Obeyesekere MN, Mechulan A, White JA, Bergin L, Khairy P, Gula LJ. Right ventricular outflow tract ventricular tachycardia ablation post-Rastelli repair. Europace. 2011;13:1050-2.

3. Khairy P, Landzberg MJ, Gatzoulis MA, Lucron H, Lambert J, Marcon F, Alexander ME, Walsh EP. Value of programmed ventricular stimulation after Tetralogy of Fallot repair: a multicenter study. Circulation. 2004;109:1994-2000.

4. Geva T, Mulder B, Gauvreau K, Babu-Narayan SV, Wald RM, Hickey K, Powell AJ, Gatzoulis MA, Valente AM. Preoperative predictors of death and sustained ventricular tachycardia after pulmonary valve replacement in patients with repaired Tetralogy of Fallot enrolled in the INDICATOR Cohort. Circulation. 2018;138:2106-15. 
5. Valente AM, Gauvreau K, Assenza GE, Babu-Narayan SV, Schreier J, Gatzoulis MA, Groenink M, Inuzuka R, Kilner PJ, Koyak Z, Landzberg MJ, Mulder B, Powell AJ, Wald R, Geva T. Contemporary predictors of death and sustained ventricular tachycardia in patients with repaired Tetralogy of Fallot enrolled in the INDICATOR cohort. Heart. 2014;100:247-53.

6. Secchi F, Lastella G, Monti CB, Barbaro U, Capra D, Zanardo M, Sardanelli F. Late gadolinium enhancement in patients with Tetralogy of Fallot: a systematic review. Eur J Radiol. 2021;136:109521.

7. Wald RM, Haber I, Wald R, Valente AM, Powell AJ, Geva T. Effects of regional dysfunction and late gadolinium enhancement on global right ventricular function and exercise capacity in patients with repaired tetralogy of fallot. Circulation. 2009;119:1370-7.

8. Babu-Narayan SV, Kilner PJ, Li W, Moon JC, Goktekin O, Davlouros PA, Khan M, Ho SY, Pennell DJ, Gatzoulis MA. Ventricular fibrosis suggested by cardiovascular magnetic resonance in adults with repaired Tetralogy of fallot and its relationship to adverse markers of clinical outcome. Circulation. 2006;113:405-13.

9. Haggerty CM, Suever JD, Pulenthiran A, Mejia-Spiegeler A, Wehner GJ, Jing L, Charnigo RJ, Fornwalt BK, Fogel MA. Association between left ventricular mechanics and diffuse myocardial fibrosis in patients with repaired Tetralogy of Fallot: a cross-sectional study. J Cardiovasc Magn Reson. 2017;19:100.

10. Oosterhof T, Mulder BJ, Vliegen HW, de Roos A. Corrected Tetralogy of Fallot: delayed enhancement in right ventricular outflow tract. Radiology. 2005;237:868-71.

11. Hunold P, Jakob H, Erbel R, Barkhausen J, Heilmaier C. Accuracy of myocardial viability imaging by cardiac MRI and PET depending on left ventricular function. World J Cardiol. 2018;10:110-8.

12. Ghonim S, Ernst S, Keegan J, Giannakidis A, Spadotto V, Voges I, Smith GC, Boutsikou M, Montanaro C, Wong T, Ho SY, McCarthy KP, Shore DF, Dimopoulos K, Uebing A, Swan L, Li W, Pennell DJ, Gatzoulis MA, Babu-Narayan SV. Three-dimensional late gadolinium enhancement cardiovascular magnetic resonance predicts inducibility of ventricular tachycardia in adults with repaired Tetralogy of Fallot. Circ Arrhythm Electrophysiol. 2020;13:e008321.

13. Cochet H, Iriart X, Allain-Nicolai A, Camaioni C, Sridi S, Nivet H, Fournier E, Dinet ML, Jalal Z, Laurent F, Montaudon M, Thambo JB. Focal scar and diffuse myocardial fibrosis are independent imaging markers in repaired Tetralogy of Fallot. Eur Heart J Cardiovasc Imaging. 2019;20:990-1003.

14. Prakash A, Powell AJ, Krishnamurthy R, Geva T. Magnetic resonance imaging evaluation of myocardial perfusion and viability in congenital and acquired pediatric heart disease. Am J Cardiol. 2004;93:657-61.
15. Preim U, Sommer P, Hoffmann J, Kehrmann J, Lehmkuhl L, Daehnert I, Gutberlet M, Grothoff M. Delayed enhancement imaging in a contemporary patient cohort following correction of Tetralogy of Fallot. Cardiol Young. 2015;25:1268-75.

16. Karur GR, Mawad W, Grosse-Wortmann L. Progressive right ventricular outflow tract fibrosis after repair of Tetralogy of Fallot. Cardiol Young. 2020;30:1366-7.

17. Radbruch A. Are some agents less likely to deposit gadolinium in the brain? Magn Reson Imaging. 2016;34:1351-4.

18. Runge VM. Critical questions regarding gadolinium deposition in the brain and body after injections of the gadolinium-based contrast agents, safety, and clinical recommendations in consideration of the EMA's pharmacovigilance and risk assessment committee recommendation for suspension of the marketing authorizations for 4 linear agents. Invest Radiol. 2017;52:317-23.

19. Fratz S, Chung T, Greil GF, Samyn MM, Taylor AM, Valsangiacomo Buechel ER, Yoo SJ, Powell AJ. Guidelines and protocols for cardiovascular magnetic resonance in children and adults with congenital heart disease: SCMR expert consensus group on congenital heart disease. J CardiovasC Magn Reson. 2013;15:51.

20. Blalock SE, Banka P, Geva T, Powell AJ, Zhou J, Prakash A. Interstudy variability in cardiac magnetic resonance imaging measurements of ventricular volume, mass, and ejection fraction in repaired Tetralogy of Fallot: a prospective observational study. J Magn Reson Imaging. 2013;38:829-35.

21. Geva T. Repaired Tetralogy of Fallot: the roles of cardiovascular magnetic resonance in evaluating pathophysiology and for pulmonary valve replacement decision support. J Cardiovasc Magn Reson. 2011;13:9.

22. Cuypers JA, Menting ME, Konings EE, Opic P, Utens EM, Helbing WA, Witsenburg M, van den Bosch AE, Ouhlous M, van Domburg RT, Rizopoulos D, Meijboom FJ, Boersma E, Bogers AJ, Roos-Hesselink JW. Unnatural history of Tetralogy of Fallot: prospective follow-up of 40 years after surgical correction. Circulation. 2014;130:1944-53.

23. Ylitalo P, Pitkänen OM, Lauerma K, Holmström M, Rahkonen O, Heikinheimo M, Sairanen H, Jokinen E. Late gadolinium enhancement (LGE) progresses with right ventricle volume in children after repair of Tetralogy of Fallot. Int J Cardiol Heart Vessel. 2014;3:15-20.

\section{Publisher's Note}

Springer Nature remains neutral with regard to jurisdictional claims in published maps and institutional affiliations.
Ready to submit your research? Choose BMC and benefit from:

- fast, convenient online submission

- thorough peer review by experienced researchers in your field

- rapid publication on acceptance

- support for research data, including large and complex data types

- gold Open Access which fosters wider collaboration and increased citations

- maximum visibility for your research: over $100 \mathrm{M}$ website views per year

At BMC, research is always in progress.

Learn more biomedcentral.com/submissions 